\title{
Spectroscopy of four-particle correlations in semiconductor microcavities
}

\author{
Salvatore Savasta, Omar Di Stefano, and Raffaello Girlanda \\ INFM and Dipartimento di Fisica della Materia e Tecnologie Fisiche Avanzate, Università di Messina Salita Sperone 31, \\ I-98166 Messina, Italy
}

\begin{abstract}
We present a theory of ultrafast time-resolved pump and probe spectroscopy in semiconductor microcavities. The numerical solutions are in excellent agreement with experimental data. An analytical study based on the Weisskopf-Wigner approximation gives physical insight into the transient optical nonlinearities of semiconductor microcavites. It is able to explain the physical origin of the unusual transient shifts and asymmetricexcitation-induced dephasing observed experimentally. This theory demonstrates that the nonperturbative regime provides a unique tool for measuring the spectrum of four-particle correlations.
\end{abstract}

DOI: 10.1103

If one or more quantum wells (QW's) are embedded in a wavelength-scale optical cavity, the light-matter interaction enters a nonperturbative regime when the collective dipole coupling rate between the QW exciton and the cavity field exceeds the relevant decay rates. ${ }^{1,2}$ This strong light-matter coupling creates two mixed photon-exciton normal modes (the cavity polaritons). Extensive studies of semiconductor microcavities (SMC's) have shown normal mode splitting of polaritons in reflection ${ }^{1}$ and emission spectra $^{3}$ and normal mode oscillations in transient optical response. ${ }^{4}$

The ultrafast nonlinear optical response of SMC's has attracted growing interest for exploring fundamental lightmatter interactions in many-particle quantum systems, ${ }^{5-11}$ as well as for future implementations of ultrafast photonic devices and of devices based on quantum correlations. Ultrafast time-resolved nonlinear spectroscopy has been shown to be a versatile tool for investigating many-body effects in QW's. ${ }^{12-14}$ In spite of many investigations and of recent progress ${ }^{7}$ in understanding the characteristic dynamic behavior of four-wave mixing (FWM) in SMC's, the coherent dynamics of the excitonic nonlinear response in the nonperturbative regime has not been fully understood. Detailed ultrafast time-resolved measurements on SMC's (Ref. 6) have shown nonlinear absorption and unusual transient Stark shifts of the polariton resonances strongly depending on the excitation energy and on the polariton branch whose origin remains unclear. A theory able to describe correctly transient optical nonlinearities of SMC's is thus required; it is also relevant for the optimization of the coherent control of polariton modes ${ }^{15}$ and for enhancing optical nonlinearities at the same time minimizing excitation-induced dephasing (EID) for applications.

In this paper we present a theory of ultrafast timeresolved pump and probe spectroscopy in the strongcoupling regime of SMC's. The theory provides physical insight into the transient optical nonlinearities of semiconductor microcavites. It is able to explain the physical origin of the transient shifts and the EID observed experimentally. It also reproduces and explains their dependence on the exciton-cavity detuning and on the pump and probe energies. One striking prediction of this theory is that when both the pump and probe are resonant with the upper polariton at zero or positive detuning it is possible to find a red- shift with cocircularly polarized pump and probe pulses. Our theoretical analysis shows that it is essential to include the full time behavior of four-particle correlations in order to reproduce and explain the ultrafast nonlinear coherent response which can be observed in pump and probe experiments.

We start from the equations for the third-order exciton polarization and cavity field describing quantum optical effects and coherent nonlinear optics in semiconductor microcavities, ${ }^{16}$ and perform the semiclassical factorization. Then we follow the procedure by Östreich et al. ${ }^{17}$ which expresses the nonlinear term coming from the Coulomb interaction as an exciton-exciton $(X-X)$ mean-field interaction plus a correlation term which is expressed as a two-exciton correlation function. Inspecting all the steps of their derivation it is possible to demonstrate that this procedure can also be applied to the nonperturbative regime of Ref. 16. Finally we include multiple scattering simply by replacing in the nonlinear sources the linear polarization and light fields with the total fields. Multiple-scattering processes are expected to be very effective in cavity-embedded QW's due to multiple reflections at the Bragg mirrors.

In order to maintain the treatment as simple as possible, we consider a specific configuration with a probe beam 1 sent along the growth axis of the microcavity (in-plane wave vector $\mathbf{k}=0)$ and a slightly tilted $(\mathbf{k} \simeq 0)$ pump beam 2 with the same circular polarization as the probe. The coherent dynamics of the photon-exciton system can be described by a set of coupled equations of motion for the probe and for the pump polarization densities $P_{j}$ and intracavity fields $E_{j}(j$ $=1,2$ ). In particular the time evolution of the probe signal is determined by the following equations:

$$
\begin{gathered}
\partial / \partial t E_{1}=-\left(\gamma_{c}+i \omega_{1}\right) E_{1}+i V P_{1}+\sqrt{g_{c}} E_{1}^{i n}, \\
\partial / \partial t P_{1}=-\left(\gamma_{x}+i \omega_{x}\right) P_{1}+i V E_{1}-i \Omega_{1}^{(N L)},
\end{gathered}
$$

where $\omega_{j}, \omega_{x}$ and $\gamma_{c}, \gamma_{x}$ are the energies and dephasing rates of cavity photons and QW excitons, respectively. $V$ is the collective dipole coupling rate, $E_{j}^{i n}$ describes the input light field, and $g_{c}$ gives the fraction of input photons passing 
the cavity mirror. The relevant nonlinear source term is given by $\Omega_{1}^{(N L)}=\Omega_{1}^{(a)}+\Omega_{1}^{(b)}$, where the first term originates from phase space filling,

$$
\Omega_{1}^{(a)}=+1 / n_{c}\left[\left|P_{2}\right|^{2} E_{1}(t)+P_{2}^{*} P_{1} E_{2}\right],
$$

being $n_{c}$ the exciton saturation density; $\Omega_{1}^{(b)}$ comes from the Coulomb interaction between electrons and can be written in the form

$$
\Omega_{1}^{(b)}=+\beta\left|P_{2}\right|^{2} P_{1}-i P_{2}^{*} \int_{-\infty}^{t} F\left(t-t^{\prime}\right) P_{1}\left(t^{\prime}\right) P_{2}\left(t^{\prime}\right) d t^{\prime},
$$

where $\beta=\left\langle 0\left|\hat{D} \hat{B}^{\dagger} \hat{B}^{\dagger}\right| 0\right\rangle$ is a measure of the mean-field $X$-X interaction being $\hat{D}=\left[\hat{B},\left[\hat{B}, \hat{V}_{C}\right]\right]\left(\hat{V}_{C}\right.$ is the Coulomb interaction operator). The equal-spin exciton-exciton correlation is contained in the retarded memory function $F(\tau)$ $=\int d \omega \mathcal{F}(\omega) e^{-i \omega t}$, where $\mathcal{F}(\omega)=\Sigma_{m}\left|\left\langle 0|\hat{D}| E_{m}\right\rangle\right|^{2} \delta\left(\omega-\omega_{m}\right)$ is the spectral density of the four-particle correlation function, with $\left|E_{m}\right\rangle$ and $\omega_{m}$ spanning the eigenstates and the corresponding continuous spectrum of energies of the fourparticle Hilbert subspace. Equation (4), which describes resonant Coulomb interactions exactly up to third order in the input fields, shows that the nonpertubative regime does not modify the retarded memory function. The strongcoupling regime affects the $X-X$ correlation only via the dynamics of the polarization densities which as is well known are dramatically modified in SMC's.

An analogous set of equations can be derived for the pump dynamics simply with the replacement $1 \rightarrow 2$ in Eqs. (1) and (2), and with the relevant nonlinear sources given by

$$
\begin{gathered}
\Omega_{2}^{(a)}=1 / n_{c}\left|P_{2}\right|^{2} E_{2}(t) \\
\Omega_{2}^{(b)}=\frac{1}{2}\left(\beta\left|P_{2}\right|^{2} P_{2}+P_{2}^{*} \int_{-\infty}^{t} F\left(t-t^{\prime}\right) P_{2}\left(t^{\prime}\right) P_{2}\left(t^{\prime}\right) d t^{\prime}\right) .
\end{gathered}
$$

We present an analytical study of the two-exciton correlation in the nonperturbative regime which is then used to understand the behavior of the numerical solutions. Let us consider a pump and probe $(j=1,2)$ ultrafast pulses with zero time delay, each centered on the lower or upper polariton energy. For the sake of simplicity, the two pulses are assumed to be sufficiently spectrally narrow to excite only one (the lower at $\omega_{L}$ or the upper at $\omega_{U}$ ) normal mode. The excited pump and probe polarizations can be written as $P_{j}$ $=\Theta(t) X_{j}(t) e^{-i \omega_{j} t}$, where $\omega_{j}$ is the energy of the polariton mode excited by the $j$ th beam and $X_{j}(t)$ are polarization amplitudes which are slowly varying functions of time as compared to $e^{-i \omega_{j} t}$ and to the decay time of the memory function $F(\tau)$. We can thus adopt the Weisskopf-Wigner approximation for the $X-X$ correlation. We obtain

$$
\Omega_{1}^{b}=\left(\Delta_{I}-i \Gamma_{I}\right)\left|P_{2}\right|^{2} P_{1},
$$

with
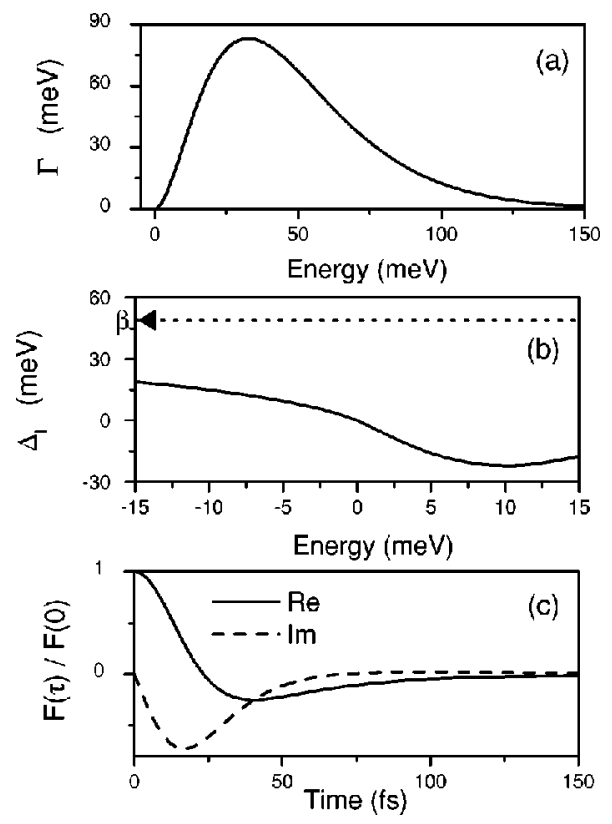

FIG. 1. (a) Spectral density of the equal-spin correlation function; parameters are given in the text. (b) Nonlinear shift as a function of energy. (c) Time behavior of the correlation function adopted for the numerical calculations.

$$
\begin{gathered}
\Gamma_{I}=\pi \mathcal{F}\left(\omega_{1}+\omega_{2}\right), \\
\Delta_{I}=\beta+\wp \int\left[\mathcal{F}(\omega) / \omega_{1}+\omega_{2}-\omega d \omega\right] .
\end{gathered}
$$

Equations (7)-(9) show that the $X-X$ correlation in SMC's determines an intensity-dependent dephasing mechanism and a renormalization of the mean-field interaction $\beta$. As expected they are proportional to the coherent exciton density generated by the pump beam $\left|P_{2}\right|^{2}$. They also have an energy dependence determined by the spectrum of four-particle correlations. In particular $\Gamma_{I}$ is directly proportional to the spectral density of the equal-spin correlation function $\mathcal{F}(\omega)$ calculated at $\omega=\omega_{1}+\omega_{2}$. We observe that by tuning the relative energy $\Delta$ between cavity and exciton resonances, and in addition exploiting the three possibilities $\omega_{1}+\omega_{2}$ $=2 \omega_{U}, \omega_{U}+\omega_{L}, 2 \omega_{L}$, it is possible to span $\Gamma_{I}$ and hence $\mathcal{F}(\omega)$ over a spectral region centered on $2 \omega_{0}$ larger than 40 meV for typical III-V SMC's. Pump and probe ultrafast measurements on SMC's can thus provide direct information on the spectrum of four-particle correlations. As expected and as confirmed by exact numerical calculations for a onedimensional (1D) semiconductor model, ${ }^{18} \mathcal{F}(\omega)$ displays strong variations within this spectral region. It consists of a continuous spectrum which has no contributions at $\omega<2 \omega_{0}$ and with a linear low-frequency behavior $\mathcal{F}(\omega) \sim \omega-2 \omega_{0}$ $>0$ followed by a high-frequency decay. Accurate correlation functions for realistic QW's are not yet available. We adopt a model correlation function introduced by Östreich and Sham ${ }^{19}$ which combines a low-frequency power law with a high-frequency decay approximated as exponential. In Fig. 1(a) the spectral density of the equal-spin correlation function $\Gamma_{I}$ is plotted by imposing a quadratic low-frequency 
behavior $^{20}$ and by using $\beta=49 /\left(\pi a^{2}\right) \mathrm{meV}, F(0)=\beta^{2} / 1.5$ ( $a$ is the two-dimensional $X$ Bohr radius). The zero of energy corresponds to the energy of two noninteracting $1 s$ excitons $\left(2 \omega_{0}=0\right)$. Figure 1 (a) together with Eq. (8) clearly predicts a suppression of the power-dependent absorption for $\omega_{1}$ $+\omega_{2}<0$. For a typical SMC in the nonperturbative regime and at zero or negative detuning, this behavior predicts a suppression of the power-dependent absorption when both the pump and the probe are tuned on the lower polariton branch $\left(\omega_{1}+\omega_{2}=2 \omega_{L}\right){ }^{21}$ This suppression, whose origin has been here clarified, is of great importance for applications; e.g., we expect it to contribute to the giant amplification of lower polaritons. ${ }^{9}$ This suppression was first observed by Baumberg et al. ${ }^{6}$ and, as we will discuss below, it has also been observed by other groups ${ }^{22-24}$ on different samples and by adopting different experimental techniques. As shown in Fig. 1(b), also the effective nonlinear susceptance $\Delta_{I}$ displays a relevant frequency dependence, passing from positive values (blueshift) at negative energies to negative values at positive energies (redshift). Furthermore, $\Delta_{I}$ is exactly zero at $\omega=0$ owing to cancellation effects imposed by a sum rule and included in the model. ${ }^{18,19}$ Figure 1(b) predicts a power-dependent shift of the polariton peaks passing from blue to red according to the sign of $\omega_{1}+\omega_{2}$. Also the observation of this puzzling energy-dependent shift has been reported. ${ }^{6}$ Figure $1(\mathrm{~b})$ shows that the effective nonlinear susceptance strongly differs from the mean-field value $\beta$ that is reached only far from resonance. In particular $\left|\Delta_{I}\right|$ is less than $20 \%$ of the $\beta$ value at energies of interest for resonant measurements.

The Weisskopf-Wigner approximation provides a very clear physical picture of correlation effects in SMC's able to explain nonlinear absorption suppression and energydependent transient shifts observed in time-resolved nonlinear measurements in SMC's. It can be easily extended to include the influence of the broadenings of the normal modes; however, it cannot describe details such as the influence of the spectral width of the light beams and ultrafast varying effects which are automatically included in our exact numerical calculations below. For these calculations we consider a realistic GaAs microcavity system with dipole coupling rate $V=2.85 \mathrm{meV}$, exciton dephasing rate $\gamma_{0}$ $=0.35 \mathrm{meV}$, and photon escape rate $\gamma_{c}=1.1 \mathrm{meV}$. The four coupled equations are numerically solved by adopting the model correlation function described above. Its time dependence is shown in Fig. 1(c).

We both use a resonant Gaussian 100 fs pump pulse with zero delay respect to the probe pulse producing a maximum exciton density $n=0.19 / \pi a^{2}$ and also, to discriminate the energy dependence of power-dependent absorption and shifts, a spectrally narrower pulse with 700 fs duration and centered on the lower or upper polariton energies. The probe is a $100 \mathrm{fs}$ pulse $10^{-4}$ weaker than the 100 fs pump pulse centered on $\omega_{0}$. In Fig. 2 we display spectra of the calculated time-resolved probe reflectivity with and without the presence of the pump beam. When the lower polariton is pumped [Fig. 2(a)], it experiences a blueshift without a corresponding shift of the upper line. Furthermore, no reduction (due to
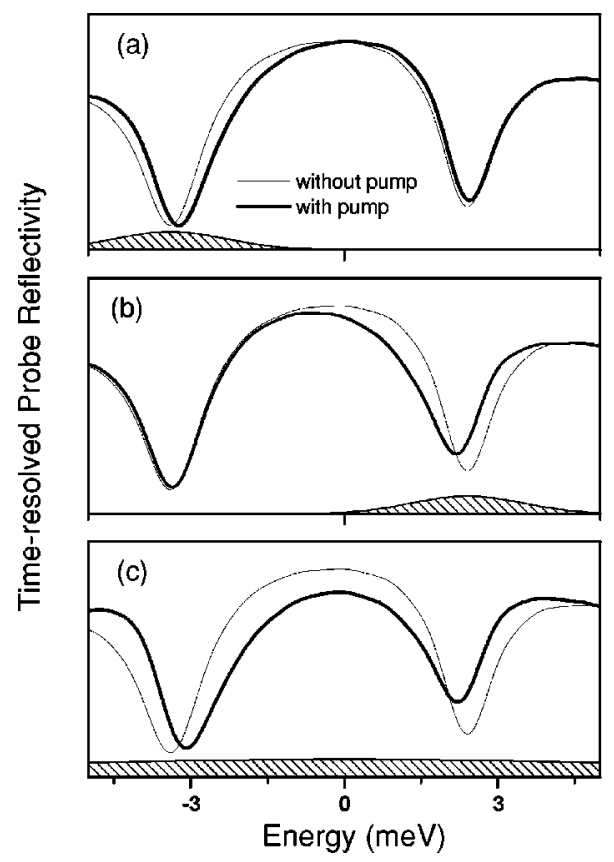

FIG. 2. Spectra of the calculated time-resolved probe reflectivity with the pump switched on (thicker line) and off. (a) and (b) Narrowband pump pulses centered on the lower and upper polariton energies. (c) Broadband pump pulse. Shaded spectra indicates the spectral shape of pump pulses.

nonlinear absorption) of the lower line is observed, while the upper resonance suffers a small bleaching. When the upper line is pumped [Fig. 2(b)] it redshifts, leaving the lower line almost unmoved. In addition a significant nonlinear absorption of the upper line and a very small nonlinear absorption of the lower line can be observed. Using the broadband 100 fs pump pulse [Fig. 2(c)], we observe an asymmetric absorption of the two lines analogous to what is observed in Fig. 2(b); a further effect is the contemporary blueshift of the lower line and redshift of the upper line. These puzzling behaviors of absorption and shifts strongly depending on the polariton branch and on the pump spectrum are determined by the $X-X$ correlation and are fully consistent with the shifts and absorption rates obtained within the Wigner-Weisskopf approximation presented above [see Figs. 1(a) and 1(b)]. By using a 1D model for correlations and a broadband pump pulse, an asymmetric nonlinear absorption close to that displayed in Fig. 2(c) has been recently found. ${ }^{25}$

Although these numerical calculations do not include the inhomogeneous broadening of excitons due to structural disorder, the agreement with Fig. 2 of Ref. 6, reporting transient probe reflection spectra with the probe arriving before and in coincidence with the pump beam, is excellent and impressive. Thus the unusual observed nonlinear effects are here reproduced and explained. The analytical study and these numerical results demonstrate that Ref. 6 provides experimental verification of the sum rule $\int \omega^{-1} \mathcal{F}(\omega) d \omega=\beta .^{18}$ Of course there is no hope of reproducing the observed highly asymmetric nonlinear absorption by replacing the correlation term in Eq. (4) with a phenomenological dephasing rate $-\sigma\left|P_{2}\right|^{2} P_{1}{ }^{7}$ Neither of these results can be described by 


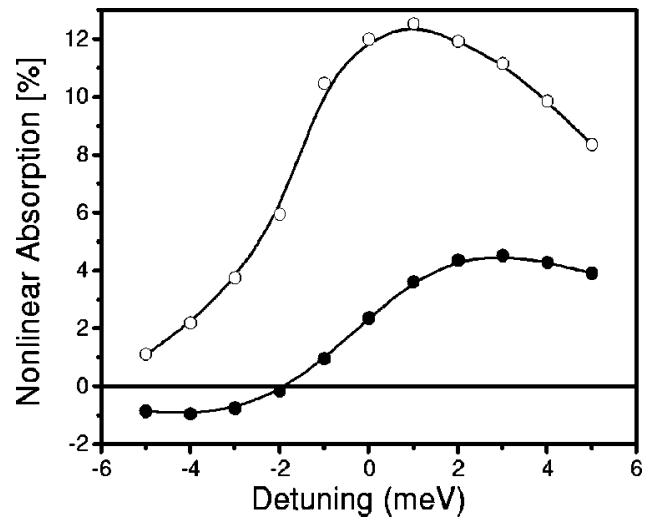

FIG. 3. Nonlinear absorption vs photon-exciton detuning $-\int \Delta T d t / \int T_{0} d t$ when pumping the lower polariton (solid circles) and the upper polariton (open circles).

connecting the polarization equation to a single-frequency biexciton density. ${ }^{13}$ Furthermore, these results clearly demonstrate a correlation-induced redshift with cocircularly polarized pump and probe pulses.

To our knowledge a systematic study of the transient Stark shift of the polariton resonances has been reported only by Ref. 6 . In a recent experiment ${ }^{15}$ demonstrating coherent control of cavity-polariton modes, with the probe pulse tuned to the upper mode and the pump pulse resonant with the lower mode $\left(\omega_{1}+\omega_{2}=0\right)$, no shift of the probe beam is observed, in agreement with this theory and with the sum rule. Instead the noticeable reduction of nolinear absorption in the lower polariton branch with respect to nonlinear absorption of the upper branch is a common feature ${ }^{22-24} \mathrm{ob}-$ served on different samples and with different experimental configurations.

In Fig. 3 we show the nonlinear absorption versus the photon-exciton detuning by displaying $-\int \Delta T d t / \int T_{0} d t, T_{0}$ being the transmittted intensity without the pump and $\Delta T$ the differential transmission determined by the pump. Absorption is always larger when pumping the upper polariton (open circles) in agreement with the Weiskoppf-Wigner approximation. When pumping the lower polariton significant absorption starts at about $\Delta=-2 \mathrm{meV}$. At negative detuning below $\Delta=-2 \mathrm{meV}$ we find a small absorption reduction. This absorption reduction originates from renormalization effects induced by the reactance part of $\Omega^{b}$ producing the shifts. Baumberg et al. ${ }^{6}$ in their Fig. 4(a) have reported nonlinear absorption versus detuning for the probe beam arriving 3 ps after the pump pulse. The overall behavior is in close agreement with Fig. 3, although absorption reduction at negative detuning was not observed. However, it was clearly observed at zero time delay [see Fig. 3(c) of Ref. 6]; it is not observed for $\tau=3$ ps because the probe delay washes out renormalization effects [see Fig. 2(b) of Ref. 6] and hence absorption reduction.

We point out that the observed long-lived effects ${ }^{6}$ with the probe arriving once where the pump pulse has completely escaped the cavity ( $\tau=3 \mathrm{ps}$ ) cannot be described within this model which does not include polariton scattering into nonradiative or poorly radiative states mainly due to structural disorder.

In conclusion we have investigated the role of fourparticle correlations in the ultrafast time-resolved nonlinear response of SMC's. We have clarified the origin of the observed very different nonlinear absorption rates of the two polariton branches and of the observed unusual transient line shifts, showing that they are a manifestation of the $X-X$ correlation. The analytical study and the numerical results here presented demonstrate that the nonperturbative regime of SMC's provides a unique tool for accessing the spectrum of four-particle correlations and provides deep insight into the transient nonlinear optical response of SMC's which should open new perspectives for engineering transient nonlinearities for ultrafast and quantum logical photonic devices.

This work was partially supported by COFIN99 Project No. 9902123352-003. We enthusiastically acknowledge discussions with J.J. Baumberg, C. Ciuti, and P. Schwendimann.
${ }^{1}$ C. Weisbuch et al., Phys. Rev. Lett. 69, 3314 (1992).

${ }^{2}$ V. Savona et al., New Aspects in Optical Properties of Nanostructures, Phase Transitions (Gordon and Breach, New York, 1998).

${ }^{3}$ R. Houdre et al., Phys. Rev. Lett. 73, 2043 (1994).

${ }^{4}$ T.B. Norris et al., Phys. Rev. B 50, 14663 (1994).

${ }^{5}$ F. Quochi et al., Phys. Rev. Lett. 80, 4733 (1998).

${ }^{6}$ J.J. Baumberg et al., Phys. Rev. Lett. 81, 661 (1998).

${ }^{7}$ H. Wang, H.Q. Hou, and B.E. Hammons, Phys. Rev. Lett. 81, 3255 (1998).

${ }^{8}$ M. Saba et al., Phys. Rev. Lett. 85, 385 (2000).

${ }^{9}$ S. Savvidis et al., Phys. Rev. Lett. 84, 1547 (2000).

${ }^{10}$ C. Ciuti et al., Phys. Rev. B 62, R4825 (2000).

${ }^{11}$ G. Khitrova et al., Rev. Mod. Phys. 71, 1591 (1999).

${ }^{12}$ P. Kner et al., Phys. Rev. Lett. 78, 1319 (1997).

${ }^{13}$ P. Kner et al., Phys. Rev. Lett. 13, 5386 (1998).

${ }^{14}$ J. Shah, Ultrafast Spectroscopy of Semiconductor and Semicon- ductor Nanostructures (Springer, Berlin, 1999).

${ }^{15}$ Y.-S. Lee et al., Phys. Status Solidi B 121, 121 (2000).

${ }^{16}$ S. Savasta and R. Girlanda, Phys. Rev. Lett. 77, 4736 (1996).

${ }^{17}$ Th. Östreich, K. Schönhammer, and L.J. Sham, Phys. Rev. Lett. 74, 4698 (1995).

${ }^{18}$ Th. Östreich, K. Schönhammer, and L.J. Sham, Phys. Rev. B 58, 12920 (1998).

${ }^{19}$ Th. Östreich and L.J. Sham, Phys. Rev. Lett. 83, 3510 (1999).

${ }^{20}$ In systems of higher dimensions than 1, phase-space arguments indicate a superlinear low-frequency behavior (Ref. 19).

${ }^{21}$ C. Ciuti et al., Phys. Rev. Lett. 84, 1752 (2000).

${ }^{22}$ F. Xudong et al., Phys. Rev. B 57, R9451 (1998).

${ }^{23}$ T. Baars et al., Phys. Rev. B 61, R2409 (2000).

${ }^{24}$ P. Borri et al., Phys. Rev. B 62, R7763 (2000).

${ }^{25}$ C. Sieh et al., Eur. Phys. J. B 11, 407 (1999). 